\title{
Optimization of Preparation Conditions for Adsorbent from Karanja Oil Seed Cake using Response Surface Methodology
}

\author{
V. N. Ganvir, \\ Dept of Petrochemical Technology,LIT Nagpur
}

\author{
Atul P. Dwivedi \\ Dept of Petrochemical Technology, LIT Nagpur
}

\begin{abstract}
:
The activated carbon was prepared by using Karanja oil seeds cakes by chemical activation using Sulphuri acid $\left(\mathrm{H}_{2} \mathrm{SO}_{4}\right)$ acid as activating agent. The effects of the Preparation variables which are activation temperature, $\mathrm{pH}$ and activation time on yield of the activated carbon were studied. Based on the central Composite design (CCD), a two factor interaction (2FI) model and a quadratic model were respectively developed to correlate the preparation variables to the adsorbent yield. From the analysis of variance (ANOVA), the most influential factor on each experimental design response was identified. The predicted adsorption yield after process optimization was found to agree satisfactory with the experimental values. The optimum conditions for preparing activated carbon from Karanja oil seed cakes were found as follows: activation temperature of $487.64{ }^{\circ} \mathrm{C}$, activation time of $2 \mathrm{~h}$ and $\mathrm{pH}$ 6.91.The characteristics and surface area of the prepared activated carbon were investigated. It was found that the specific surface area of activated was (about 343 $\mathrm{m} 2 / \mathrm{g}$ ). The XRD analysis have been done.
\end{abstract}

\section{Keywords:}

activated carbon, karanja oil seed cakes, surface area and $\mathrm{XRD}$, central Composite design optimization.

\section{INTRODUCTION:}

Today many agricultural byproducts such as walnut shell, coconut shell, cherrystone, Apricot stone, nuts, grape seeds, eucalyptus, olive and peach stones, sugar cane bagasse and oil palm trunks have been to be suitable precursors owing to their high carbon and low ash contents for the preparation of activated carbon [1].

A challenge in activated carbon production is to produce very specific carbons which are suitable for certain applications. The most important characteristic of an activated carbon is its adsorption capacity which is highly influenced by the preparation conditions. In assessing the effect of treatments on quality attributes, the use of an adequate experimental design is particularly important. Response surface methodology (RSM) has been found to be a useful tool to study the interactions of two or more variables [2]. Optimization of experimental conditions using RSM was widely applied in various processes; however, its application in activated carbon production is very rare. Some of the previous studies found in applying RSM in preparation of activated carbons were using precursors such as olive-waste cakes, Luscar char and Turkish lignite. As far as known, no study has been done on preparation of activated carbon from karanja oil seed cake with chemical activation method consisting of Sulphuri acid (H2SO4) treatment and by using RSM approach.
Their application in industry includes removing organic and inorganic pollutants from drinking water, industrial wastewater treatment, decolorizing of syrups and purification of air and pharmaceutical product [3]. Because of their wide usage in industry, the demand of the activated carbon is increasing year by year. Commercial activated carbon is quite expensive. As such industry now seeks for the cheapest activated carbon derive from agriculture waste or residuals. The residuals can be seed cakes rice husk, sawdust or other carbonaceous material. The raw material were processed and optimized to obtain excellent adsorptive properties.

\section{MATERIAL AND METHOD:}

\subsection{Preparation of activated carbon:}

Karanja oil seed cake used for preparation of activated carbon was obtained locally. The cake was first washed to remove the dirt and the dust from the surface and then dried overnight in the oven at $105^{\circ} \mathrm{C}$. The dried cake was then digested using Sulphuri acid (H2SO4) and kept in the oven for 24 hrs. For digestion to take place completely. The digested cake is then washed to bring the wash water $\mathrm{pH}$ to 6-7. The washed cake is then kept in the oven for $2 \mathrm{hr}$ to remove moisture from it at $110^{\circ} \mathrm{C}$. The dried cake is then carbonized at $500^{\circ} \mathrm{C}$ in the muffle furnace. The carbonized material is then crushed to desired mesh size.

\subsection{Design of experiment:}

The parameters for adsorbent preparation are studied with a standard response surface methodology (RSM). In this, central composite design (CCD) was used. This method is suitable for fitting the quadratic surface and it helps to optimize the effective parameters with a minimum number of experiments, and also to analyze the interaction between the parameters [4]. Generally the CCD consist of a $2^{\mathrm{n}}$ factorial runs with $2 \mathrm{n}$ axial runs and $\mathrm{n}_{\mathrm{c}}$ centers runs (six replicates) The variable studied 1) $\mathrm{x} 1$ : reaction temperature 2) $\mathrm{x} 2 \mathrm{pH}$ of the material.

Thus total no. of runs $(\mathrm{N})$ required is 14

$\mathrm{N}=2^{\mathrm{n}}+2 \mathrm{n}+\mathrm{n}_{\mathrm{c}}=2^{2}+2 * 2+6=$

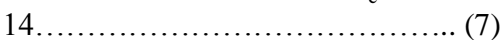

The center points use to determine the experimental error and the reproducibility of the data. The variable are coded to the ($1,1)$ intervals where the low and high levels are coded as -1 and 1 respectively. The axial points are located at $( \pm \alpha, 0),(0$, $\pm \alpha$ ) where $\alpha$ is the distance of the axial points from centre and makes the design ratable. The value was fixed at 1.682(ratable). The response was adsorbent yield (y). The correlation was used to develop an empirical model that correlated the response to the adsorbent yield variable using equations.

The equation in coded form is givens as:- 
$\mathrm{Y}=72.06-4.97 * \mathrm{X}_{1}-0.14 * \mathrm{X}_{2}-1 * \mathrm{X}_{1} * \mathrm{X}_{2}-18.11 * \mathrm{X}_{1}{ }^{2}+3.2 *$

$\mathrm{X}_{2}^{2}$....... (1)

The actual equation is:-

$\mathrm{Y}=-54.48+3.18 * \mathrm{X}_{1}-189.99 * \quad \mathrm{X}_{2}-0.029 * \quad \mathrm{X}_{1} * \quad \mathrm{X}_{2}-3.22 *$ $\mathrm{X}_{1}^{2}+15.79 * \mathrm{X}_{2}^{2}$

\subsection{Experimental Design Matrix and} Results:-

\begin{tabular}{|c|c|c|c|}
\hline sr no. & temp & ph & yield \% \\
\hline 1 & 400 & 6.9 & 72 \\
\hline 2 & 475 & 6.45 & 72.34 \\
\hline 3 & 581.0660172 & 6.45 & 20 \\
\hline 4 & 550 & 6 & 62 \\
\hline 5 & 475 & 6.45 & 71.2 \\
\hline 6 & 475 & 5.813603897 & 70 \\
\hline 7 & 475 & 6.45 & 72.25 \\
\hline 8 & 400 & 6 & 70 \\
\hline 9 & 475 & 6.45 & 71.33 \\
\hline 10 & 550 & 6.9 & 60 \\
\hline 11 & 475 & 7.086396103 & 69.23 \\
\hline 12 & 475 & 6.45 & 73.2 \\
\hline 13 & 368.9339828 & 6.45 & 34 \\
\hline
\end{tabular}

The analysis is done using DESIGN 8 EXPERT software which gave us the 3D curve of two parameter against the response as shown below:-

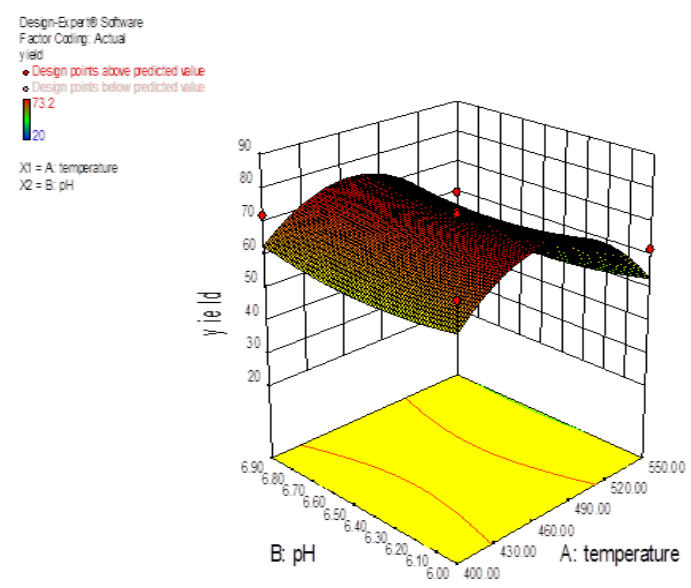

\section{RESULTS AND DISCUSSION:}

The results obtained can be studied to get optimized conditions for the response vs parameters. And one can also find the approximate values of the response for the required set of parameters. In this case we got the optimum response $=75.23 \%$ for the conditions of the parameters temp $=487.01^{\circ} \mathrm{c}$ and $\mathrm{pH} 6.91$.

\subsection{Surface Properties:}

3.1.1 Surface area of the adsorbents $=343 \mathrm{~m}^{2} / \mathrm{g}$

3.1.2 XRD analysis:

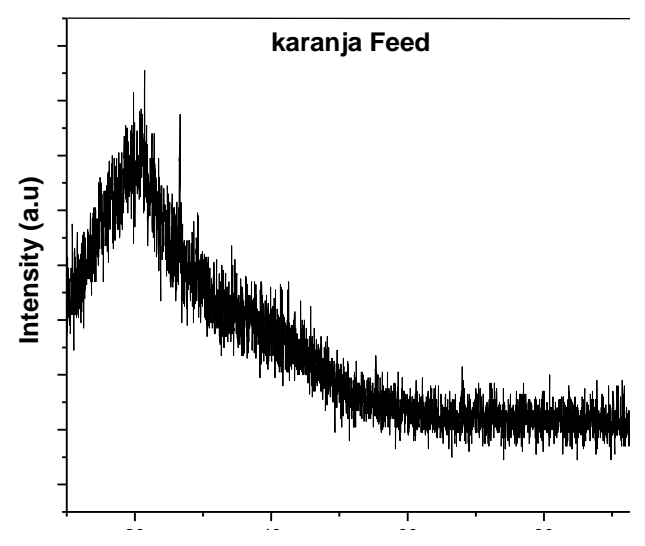

Fig 1(XRD of Karanja feed)

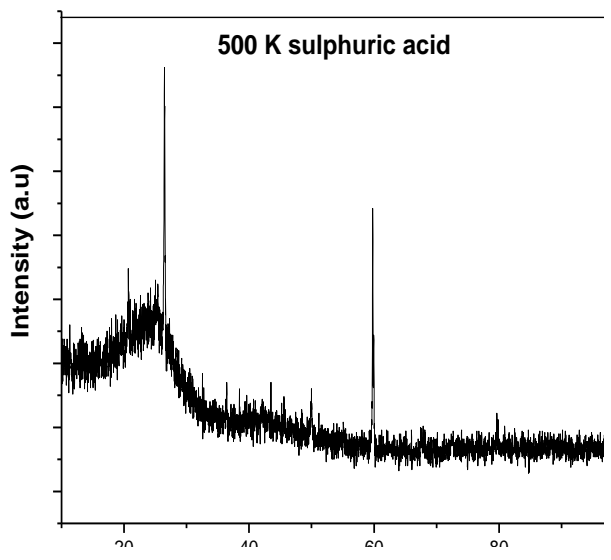

Fig 2(XRD of carbonized karanja)

Fig 1 and Fig 2 shows the phase change from amorphous to crystalline form by the change in peak height.

\section{CONCLUSION:}

The optimization of the prepration variables has been studied and the optimum response (yield) $=75.23 \%$ for the conditions of the parameters temp $=487.01 \mathrm{oc}$ and $\mathrm{pH}$ 6.91. has been found.

\section{REFERENCES:}

[1] V.N.Ganvir, M.L.Meshram, Atul P. Dwivedi, "Preparation of adsorbent from Karanja oil seeds cakes and its characterization". International Journal of computer application (EFITRA-2012 Proceedings) April 2012.

[2] F. Karacan, U. Ozden, S. Karacan, Optimization of manufacturing conditionsfor activated carbon from Turkish lignite by chemical activation using response surface methodology, Appl. Therm. Eng. 27 (2007) 1212-1218.

[3] V.H.Chipofya and G.L. McConnachie "Characterization of activated carbon produced from agricultural waste materials for taste and odor removal in drinking water supplies", 1st WARFSA/WaterNet Symposium: Sustainable Use of Water Resources, Maputo, 1-2 November 2000.

[4] Dr. Y.C. Bhattacharyulu, V. N. Ganvir, Miss Nisha Balani \& Mr. Akash Parate "optimization of biodiesel production using response surface methodology". IE (2011) 222-234. 